\section{Transcriptional activities of DUX4 fusions in B-cell acute lymphoblastic leukemia}

DUX4 fusions are novel oncogenes in a subset of $\mathrm{B}$-cell acute lymphoblastic leukemia (B-ALL) clinical cases, generally in older children and adolescent patients. ${ }^{1}$ DUX4 fusion transcripts are generated from insertions of wildtype (WT) DUX4, mainly into the IGH locus. ${ }^{1.4}$ Notably, the translocation replaces the $3^{\prime}$ end of the WT DUX4 coding region with a fragment of IGH or another gene, producing DUX4 out-of-frame fusion proteins devoid of the C terminus of WT DUX4 protein. Importantly, the introduction of DUX4-IGH into a mouse pro-B cell results in defective differentiation of $B$ cells, and ultimately leukemic transformation.
WT DUX4 is a transcription factor with a doublehomeobox domain, coded in a macrosatellite repeat region called D4Z4 in subtelomeric regions of chromosomes 4 and 10. In normal physiology, WT DUX4 is expressed in germ cells, such as testis, while its expression is usually epigenetically repressed in somatic tissues, ${ }^{5}$ and it plays a critical role in embryonic cleavagespecific transcriptional programs. ${ }^{6-8}$

To date, insertion of the DUX4 gene has been implicated as the leading event in the pathogenesis of B-ALL carrying DUX4 fusions. The resultant DUX4 fusions possess transforming abilities, even though WT DUX4 is presumed to be pro-apoptotic. ${ }^{5}$

To investigate the transcriptional targets of DUX4IGH, we first performed ChIP-seq with anti-DUX4 antibody and anti-H3K4me3 antibody, using NALM6, a
A

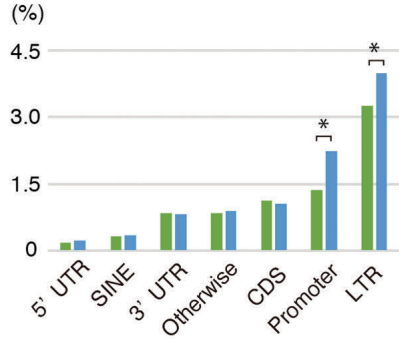

C

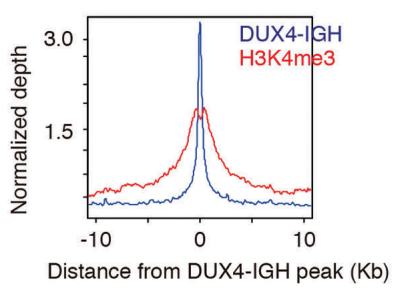

$(\%)$

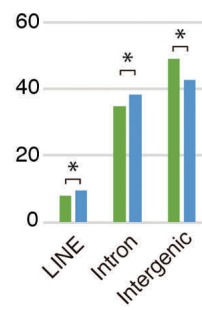

B

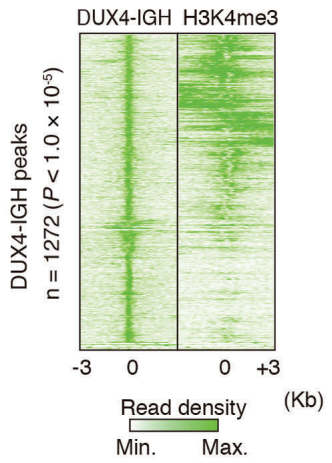

DUX4-IGH (NALM6)

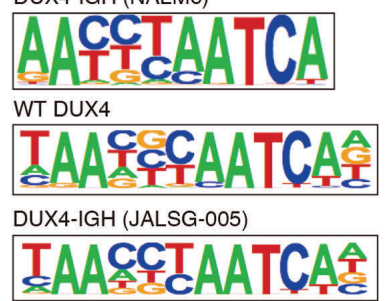

$P=1 \mathrm{E}-155$

$46.38 \%$ of targets

$4.69 \%$ of background

$P=1 \mathrm{E}-16184$

$33.67 \%$ of targets

$3.04 \%$ of background

$P=1 \mathrm{E}-6218$

$52.03 \%$ of targets

$1.25 \%$ of background

E
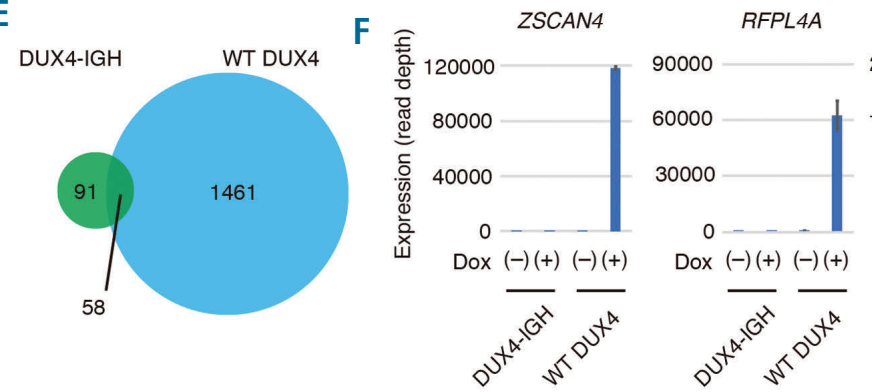

DUXA

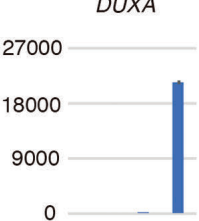

ox $(-)(+)(-)(+)$

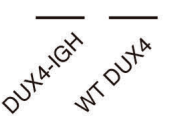

G

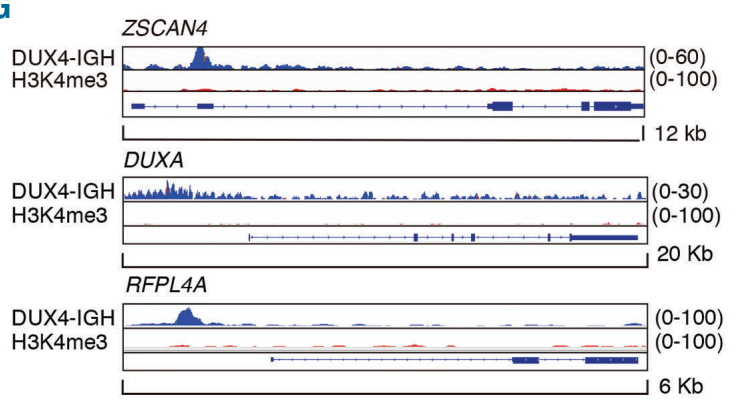

$\mathrm{H}$

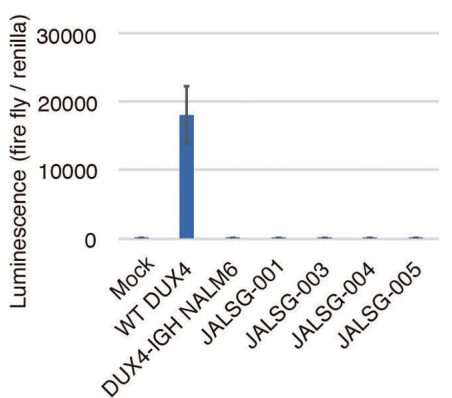

Figure 1. Genome-wide DUX4-IGH binding in NALM6 cells. (A) The proportion of mapped regions in the human genome for reads obtained by chromatin immunoprecipitation coupled with sequencing (ChIP-seq) against DUX4-IGH in NALM6 cells (right, blue). The proportion of the respective regions within the human genome is shown on the left for comparison (green). ${ }^{*} P<0.0001$, test of proportion. (B) Density heat maps of DUX4-IGH (left) and trimethylated lysine residue at position four of histone $\mathrm{H} 3$ (H3K4me3) (right). (C) The average binding densities around DUX4-IGH peaks are shown: blue, DUX4-IGH; red, H3K4me3. (D) De novo motif-discovery analysis using HOMER software. DUX4-IGH (endogenous) in NALM6 (upper panel), WT DUX4 (ectopic, Hendrickson et al. 2017) in induced pluripotent stem cells (middle panel), DUX4-IGH (JALSG005type, ectopic) in Reh cells (lower panel) (E) Venn diagram showing the number of genes upregulated by overexpression of DUX4-IGH (green) or wild-type (WT) DUX4 (light blue) in NALM6 cells. (F) Expression of the representative target genes of WT DUX4 in NALM6 cells, in which expression of DUX4-IGH or WT DUX4 was induced by doxycycline (Dox). (G) Mapped reads at the ZSCAN4, DUXA, RFPL4A locus obtained with chromatin immunoprecipitation coupled with sequencing (ChIP-seq) against DUX4-IGH or trimethylated lysine residue at position four of histone $\mathrm{H3}$ (H3K4me3) in NALM6 cells. $(\mathrm{H})$ Luciferase assay using the reporter plasmid containing ZSCAN4 promoter. LINE: long interspersed nuclear element; LTR: long terminal repeat; FDR: false discovery rate. 
B-cell acute lymphoblastic leukemia cell line carrying DUX4-IGH fusion. We detected 1,272 peaks ( $P$-value cutoff: $1.0 \times 10^{-5}$ ) with ChIP-seq against endogenous DUX4IGH. Promoter, intronic, long terminal repeat, LINE regions were increased in the DUX4-IGH binding sites (Figure 1A), consistent with the previous report which showed increased binding of WT DUX4 in long terminal repeat regions. When the reads marked by $\mathrm{H} 3 \mathrm{~K} 4 \mathrm{me} 3$ were aligned against read peaks of DUX4-IGH, they were preferentially clustered toward the center of the DUX4IGH peaks, indicating that $\mathrm{H} 3 \mathrm{~K} 4 \mathrm{me} 3$ colocalized with DUX4-IGH (Figures $1 \mathrm{~B}$ and 1C). Motif analysis using DUX4-IGH binding regions identified a motif that was almost identical to that recognized within WT DUX4 binding regions in human myoblast and embryonic cells (Figure 1D, top), which was also recapitulated by reanalysis of the published data on ChIP-seq of WT DUX4 in induced pluripotent stem (iPS) cells (Figure 1D, middle). Furthermore, ChIP-seq using anti-DUX4 antibody in Reh cells ectopically expressing another type of DUX4-IGH identified in a clinical sample (JALSG005) ${ }^{1}$ indicated a similar motif (Figure 1D, lower). In every case, only the DUX4 motif was statistically significant, suggesting the absence of a transcription factor that colocalizes with DUX4-IGH.

While DUX4-IGH has the capacity to transform pro-B cells, induction of WT DUX4 causes cell death in somatic tissues. To investigate the functional difference between DUX4-IGH and WT DUX4, we compared the genes detected with our ChIP-seq and genes described in a previous report of ChIP-seq against WT DUX4 in iPS cells. Ninety-seven percent $(1057 / 1082)$ of genes detected in
A

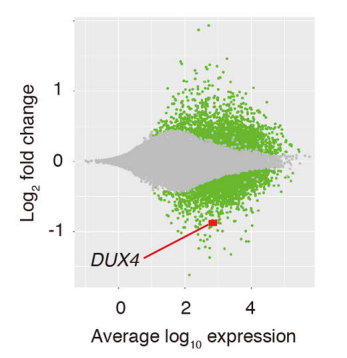

B

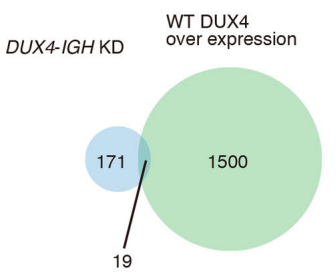

C

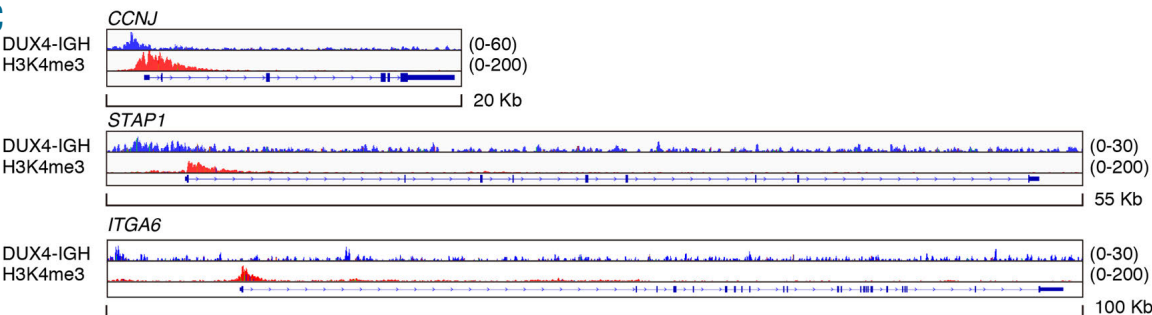

D
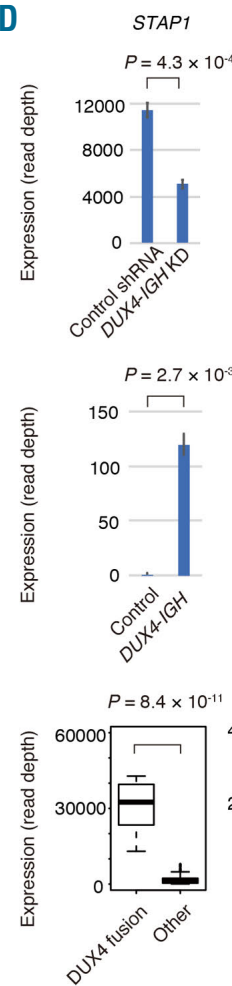

CCNJ
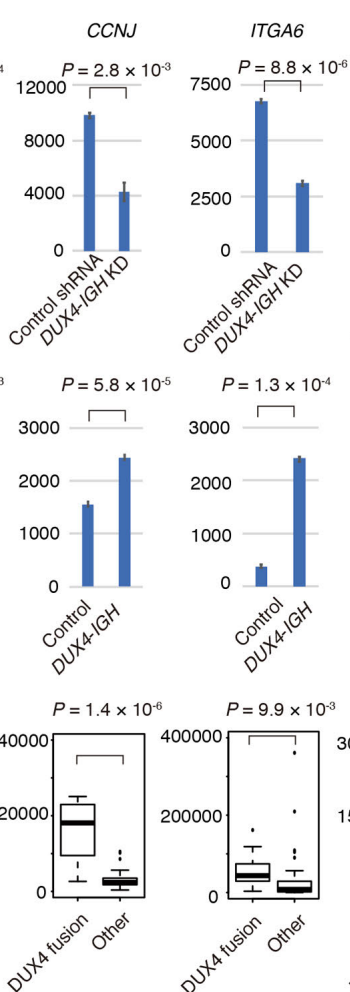

$P=9.9 \times 10^{-3}$

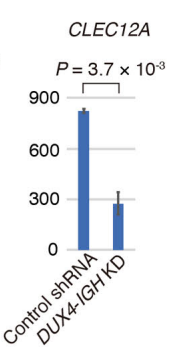

$P=6.5 \times 10^{-2}$
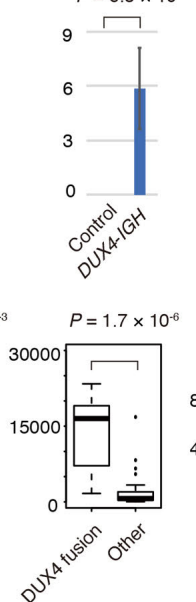

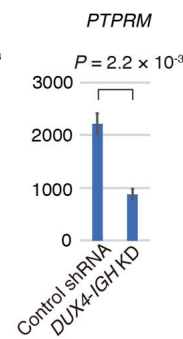

DDIT4L

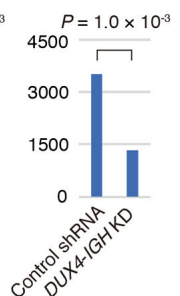

$P=7.4 \times 10^{-4}$
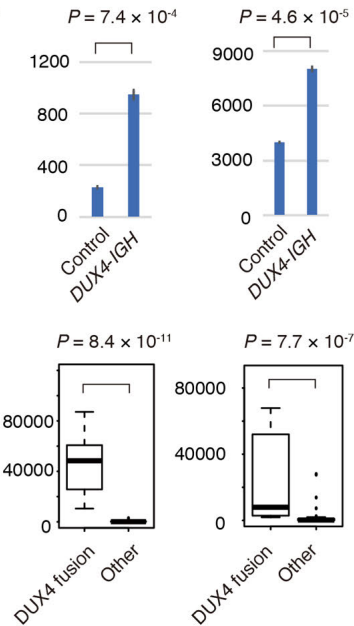

Figure 2. Knockdown of DUX4 IGH with short hairpin RNA. (A) MA plot showing the change in the transcripts with DUX4-IGH knockdown. Genes with a significant change $(P<0.05)$ in expression are plotted in green, and DUX4 is plotted in red. (B) Venn diagram showing the number of genes underrepresented in DUX4-IGH knockdown in NALM6 cells (light blue) or genes whose expression was upregulated by overexpression of wild-type (WT) DUX4 in NALM6 cells (light green). (C) Mapped reads at the CCNJ, STAP1, or ITGA6 locus obtained with chromatin immunoprecipitation coupled with sequencing (ChIP-seq) against DUX4-IGH (blue) or trimethylated lysine residue at position four of histone $\mathrm{H} 3$ (H3K4me3, red) in NALM6 cells. (D) Expression of representative genes in NALM6 infected with control shRNA or shRNA against DUX4-IGH (upper panel). Expression of the genes in Reh infected with control vector or DUX4-IGH expression vector (middle panel). Expression of the genes in clinical samples of B-ALL with or without DUX4 fusion (lower panel). 
our ChIP-seq against DUX4-IGH were included among the detected genes in the ChIP-seq analysis using iPS cells, suggesting that DNA binding motif recognition by DUX4-IGH was not altered compared with that by WT DUX4 (Online Supplementary Figure S1).

To identify the genes regulated by DUX4-IGH and WT DUX4, we introduced inducible vectors encoding DUX4IGH (NALM6-type) or WT DUX4 into NALM6 cells and Reh cells. In NALM6 cells, the expression of 149 genes was upregulated $(>1.5$-fold, an adjusted $P$-value of $<0.05$ ) by DUX4-IGH, while 1519 genes were upregulated by WT DUX4 ( $>1.5$-fold, an adjusted $P$-value of $<0.05)$ (Figure 1E). Despite the large overlap between the genes bound by DUX4-IGH and those bound by WT DUX4, only a small number of genes were upregulated by both DUX4-IGH and WT DUX4. Well-known targets of WT DUX4, such as ZSCAN4, DUXA, and RFPL4A, were prominently upregulated by the overexpression of WT

A
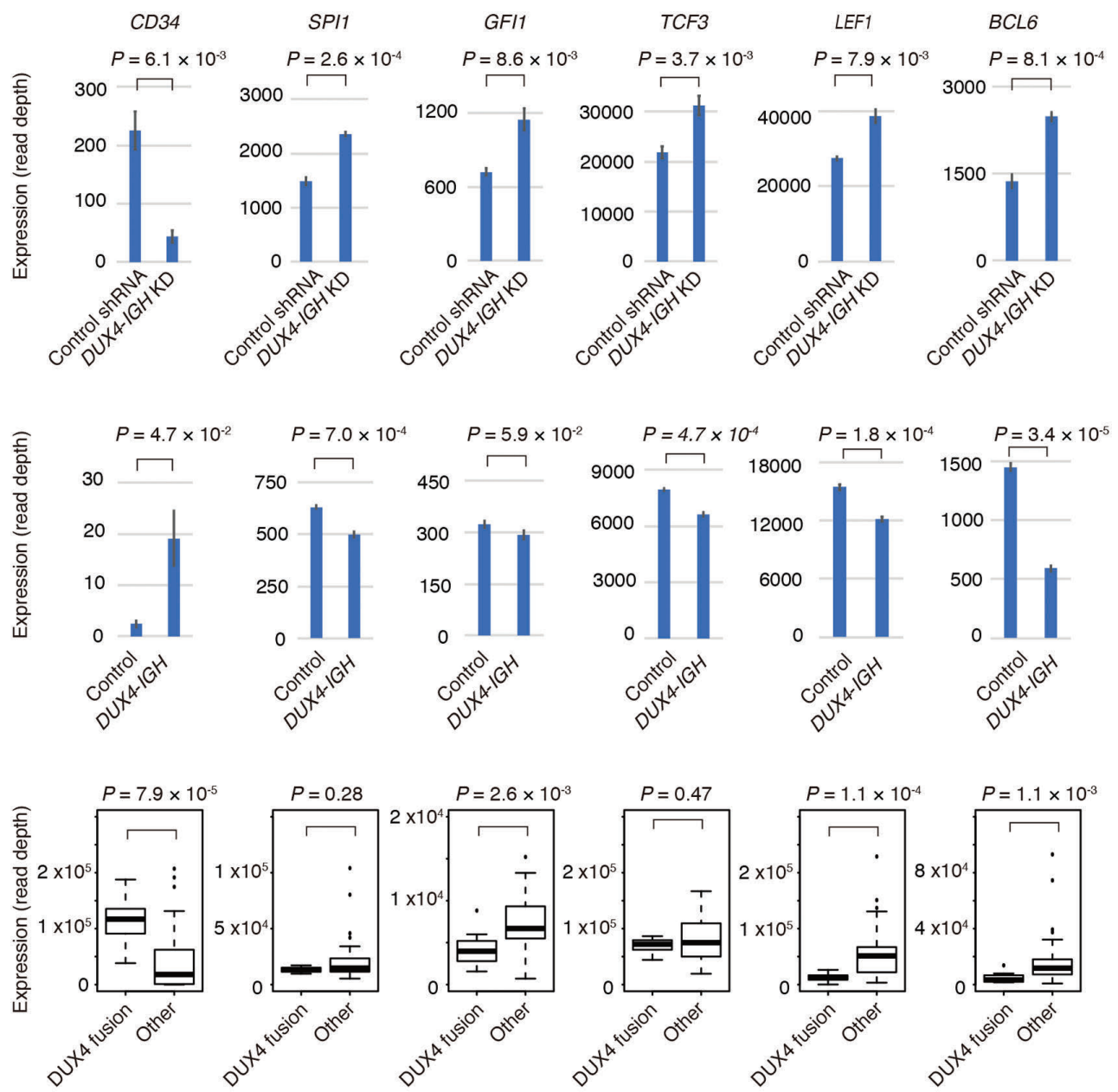

B

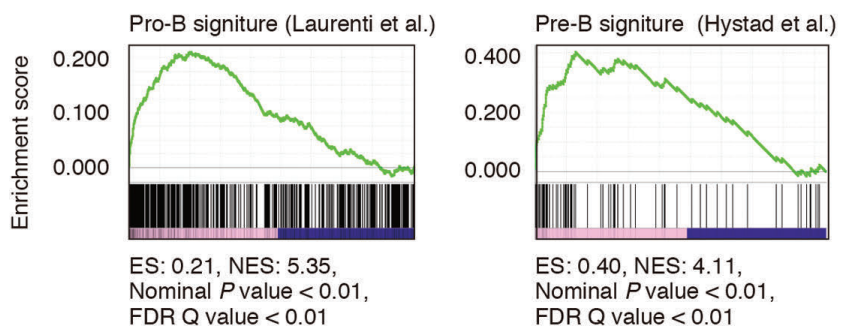

Figure 3. Genes associated with hematopoietic cell function regulated by DUX4-IGH. (A) Expression of representative genes related to hematopoietic differentiation in NALM6 cells infected with control short hairpin RNA (shRNA) or shRNA against DUX4-IGH (upper panel). Expression of the genes in Reh infected with control vector or DUX4-IGH expression vector (middle panel). Expression of the genes in clinical samples of B-ALL with or without DUX4 fusion (lower panel). (B) Gene set enrichment analysis of the underrepresented or overrepresented genes in NALM6 cells with DUX4-IGH knockdown. ES: enrichment score; NES: nominal enrichment score; FDR: false discovery rate. 
DUX4, but not by DUX4-IGH (NALM6-type) (Figure 1F). Similarly, these genes were not upregulated by overexpression of DUX4-IGH in Reh cells. H3K4me3 peaks were not detected near these genes with ChIP-seq in NALM6 cells (Figure 1G), confirming that these genes were not regulated by DUX4-IGH. Moreover, luciferase assay of DUX4-IGH or WT DUX4 using ZSCAN4 promoter indicated decreased transcriptional activity of various types of DUX4-IGH fusion ${ }^{1}$ compared with that of WT DUX4 (Figure 1H). These results suggested that the transactivation ability of DUX4-IGH is attenuated compared with that of WT DUX4. This is in good agreement with a previous report describing that DUX4-s, a short variant of DUX4 that maintains the $\mathrm{N}$-terminal doublehomeobox domain but lacks the C-terminal transactivation domain, loses transactivation ability, ${ }^{9}$ possibly through an inability to bind EP300.

We previously showed that the knockdown of DUX4-IGH with shRNA leads to decreased proliferation of NALM6 cells, which can be rescued by the introduction of shRNA-resistant DUX4 fusion complementary DNA. To investigate the genes regulated by DUX4-IGH, NALM6 cells were infected with a retroviral vector expressing shRNA against DUX4-IGH or control shRNA. The expression level of DUX4-IGH was found to be decreased to $52 \%$ with the shRNA. We identified 279 significantly overrepresented genes (DUX4-IGH shRNA/control shRNA $>1.5$, an adjusted $P$-value of $<0.05)$ and 190 significantly underrepresented genes (control shRNA/DUX4-IGH shRNA >1.5, an adjusted $P$-value of $<0.05)$ (Figure $2 \mathrm{~A}$ and Online Supplementary Table). Only $1.2 \%(19 / 1519)$ of genes upregulated by the induction of WT DUX4 in NALM6 cells in the prior experiment overlapped with genes underrepresented in NALM6 cells with DUX4-IGH knockdown (Figure 2B).

The genes underrepresented in NALM6 cells with DUX4-IGH knockdown were supposed to comprise genes positively regulated by DUX4-IGH. Only $14 \%$ (26/190) of them were associated with DUX4-IGHbound regions identified in ChIP-seq, indicating that the majority of the genes dysregulated by DUX4-IGH knockdown were not direct transcriptional targets of DUX4-IGH. Among the direct target genes of DUX4IGH, 93\% (24/26) were marked with H3K4me3, indicating that DUX4-IGH substantially contributed to transcriptional activity in NALM6 cells. Direct target genes with the largest fold change in expression with DUX4$I G H$ knockdown include STAP1, CCNJ, and ITGA6 (Figure 2C and 2D), whereas indirect target genes with the largest fold change in expression include CLEC12A, PTPRM, and DDIT4L. STAP1, CCNJ, and ITGA6 were also bound by DUX4-IGH (JALSG005-type) (Online Supplementary Figure S2). In Reh cells, overexpression of DUX4-IGH induced expression of these direct and indirect target genes (Figure 2D). In the RNA-seq from JALSG B-ALL clinical samples, we observed significantly higher expression of these genes in B-ALL cases with DUX4 fusion, compared with other types of B-ALL cases (Figure 2D), further validating the specific transcriptional activity of DUX4-IGH. Furthermore, the same pattern of expression was recapitulated regarding the other genes reported to be distinctly expressed in a cluster of leukemia with ERG deletion, ${ }^{10}$ such as AGAP1, CHST2, GPR155, and PAM (Online Supplementary Figure S3). Taken together, DUX4-IGH exerts the transcriptional activity in forming the characteristic expression signature of B-ALL carrying DUX4-fusions, directly and indirectly. To investigate the role of these genes in the survival of NALM6 cells, we performed knockdown of these genes using shRNA, focusing especially on STAP1, CCNJ, CLEC12A. However, single knockdown of these genes did not inhibit the growth of NALM6 cells (Online Supplementary Figure S4).

CD34 was underrepresented in NALM6 cells with DUX4-IGH knockdown, and was overrepresented in Reh cells with DUX4-IGH overexpression. CD34 is also highly expressed in clinical samples with DUX4 fusion. Interestingly, genes overrepresented in NALM6 cells with DUX4-IGH knockdown included those associated with hematopoietic cell differentiation, and exhibit a low expression level specifically in clinical samples with DUX4 fusion (Figure 3A). None of these genes were bound by DUX4-IGH, according to our ChIP-seq data. Expression levels of these genes were moderately repressed in Reh cells and NALM6 cells by DUX4-IGH overexpression (Online Supplementary Figure S5). Furthermore, these genes include those involved in early B-lymphoid development, such as TCF3 (E2A) and SPI1 (PU.1). TCF3 knockout mice exhibited blocked maturation of B cell. ${ }^{11}$ SPI1-deficient mice also failed to generate the earliest lymphoid progenitors. ${ }^{12}$ In addition, transcription factors regulated through various development stages of B lymphocytes, such as GFI1, LEF1, and BCL6 were significantly decreased in B-ALL cells with DUX4 fusions. These genes also play important roles in phases before lymphoid lineage priming.

To clarify the effect of DUX4-IGH knockdown on B-cell differentiation, we performed GSEA using the datasets from Hystad et al. ${ }^{14}$ and Laurenti et al. ${ }^{15}$ GSEA revealed that genes associated with pro-B cells, pre-B cells (Figure $3 \mathrm{~B}$ ), and multilineage progenitor cells were enriched in the genes overrepresented in NALM6 cells with DUX4-IGH knockdown, while genes associated with megakaryocyte-erythroid progenitor cells and common myeloid progenitor cells were enriched in the genes underrepresented in NALM6 cells with DUX4-IGH knockdown (Online Supplementary Figure S6). This result indicated that DUX4-IGH enables progenitor cells to retain an immature status, and knockdown of DUX4IGH drives NALM6 cells toward B-cell differentiation, which is compatible with our previous results in vivo demonstrating that murine B-lymphocyte differentiation is abrogated by the expression of DUX4-IGH.

In conclusion, we revealed that DUX4-IGH exhibited distinct transcription-inducing activity compared with WT DUX4, which was associated with the defective differentiation of $B$ cells. Further understanding of the mechanism of transcriptional network perturbation by DUX4-IGH should provide clues for a treatment strategy for B-ALL carrying DUX4 fusions.

Yosuke Tanaka, Masahito Kawazu, ${ }^{2}$ Takahiko Yasuda, ${ }^{1,3}$ Miki Tamura, ${ }^{1}$ Fumihiko Hayakawa, ${ }^{4}$ Shinya Kojima,

Toshihide Ueno, ${ }^{1}$ Hitoshi Kiyoi, ${ }^{4}$ Tomoki Naoe and Hiroyuki $M_{\text {Mano }}^{1,6}$

Departments of Cellular Signaling and ${ }^{2}$ Medical Genomics, Graduate School of Medicine, The University of Tokyo; ${ }^{3}$ Division of Biological Information Analysis, Department of Clinical Research Management, Clinical Research Center, National Hospital Organization Nagoya Medical Center, Aichi; ${ }^{4}$ Department of Hematology and Oncology, Nagoya University Graduate School of Medicine, Aichi; ${ }^{5}$ National Hospital Organization Nagoya Medical Center, Aichi and ${ }^{6}$ National Cancer Center Research Institute, Tokyo, Japan

Funding: this work was supported in-part by Grant-in-Aid for Scientific Research (KAKENHI) from the Japan Society for the

Promotion of Science to YT (17J10130), and by grants for the Project for Cancer Research and Therapeutics Evolution (P-CREATE) 
LETTERS TO THE EDITOR

(17cm0106525h0002 to TY), for Leading Advanced Projects for Medical Innovation (LEAP) (17am0001001 h0004 to HM) and for Practical Research for Innovative Cancer Control 17ck0106331 h0001 to FH, and 17ck0106167h0003 to HM) from the Japan Agency for Medical Research and Development.

Correspondence: mkawz-tky@umin.ac.jp doi:10.3324/haematol.2017.183152

Information on authorship, contributions, and financial \& other discosure was provided by the authors and is available with the online version of this article at www. haematologica.org.

References

1. Yasuda T, Tsuzuki S, Kawazu M, et al. Recurrent DUX4 fusions in B cell acute lymphoblastic leukemia of adolescents and young adults. Nat Genet. 2016;48(5):569-574.

2. Lilljebjorn $\mathrm{H}$, Henningsson $\mathrm{R}$, Hyrenius-Wittsten $\mathrm{A}$, et al. Identification of ETV6-RUNX1-like and DUX4-rearranged subtypes in paediatric B-cell precursor acute lymphoblastic leukaemia. Nat Common. 2016;7:11790.

3. Lieu YF, Wang BY, Zhang WN, et al. Genomic profiling of adult and pediatric B-cell acute lymphoblastic leukemia. BioMedicine. 2016; 8:173-183.

4. Chang J, McCastlain K, Yoshihara H, et al. Deregulation of DUX4 and ERG in acute lymphoblastic leukemia. Nat Genet. 2016; 48(12):1481-1489.

5. Gens LN, Yo Z, Snider L, et al. DUX4 activates germline genes, retroelements, and immune mediators: implications for facioscapulohumeral dystrophy. Lev Cell. 2012;22(1):38-51.

6. Hendrickson PG, Doris JA, Grow EJ, et al. Conserved roles of mouse DUX and human DUX4 in activating cleavage-stage genes and MERVL/HERVL retrotransposons. Nat Genet. 2017;49(6):925934.
7. Whiddon JL, Langford AT, Wong CJ, Zhong JW, Tapscott SJ. Conservation and innovation in the DUX4-family gene network. Nat Genet. 2017;49(6):935-940.

8. De Taco A, Planet E, Coluccio A, Vern S, Dur J, Trono D. DUX-family transcription factors regulate zygotic genome activation in placental mammals. Nat Genet. 2017;49(6):941-945.

9. Chi SH, Gearhart MD, Gui Z, et al. DUX4 recruits p300/CBP through its C-terminus and induces global H3K27 acetylation changes. Nucleic Acids Res. 2016;44(11):5161-5173.

10. Harvey RC, Mullighan CG, Wang X, et al. Identification of novel cluster groups in pediatric high-risk B-precursor acute lymphoblastic leukemia with gene expression profiling: correlation with genomewide DNA copy number alterations, clinical characteristics, and outcome. Blood. 2010;116(23):4874-4884.

11. Dias S, Manson R, Gurbuxani S, Sigvardsson M, Re BL. E2A protens promote development of lymphoid-primed multipotent progenitors. Immunity. 2008;29(2):217-227.

12. Iwasaki $\mathrm{H}$, Somoza C, Shigematsu $\mathrm{H}$, et al. Distinctive and indispensable roles of PU.1 in maintenance of hematopoietic stem cells and their differentiation. Blood. 2005;106(5):1590-1600.

13. Spooner CJ, Chen JX, Pujadas E, Lasso P, Singh H. A recurrent network involving the transcription factors PU.1 and Gil orchestrates innate and adaptive immune cell fates. Immunity. 2009;31(4):576586.

14. Hystad ME, Myklebust JH, Bo TH, et al. Characterization of early stages of human B cell development by gene expression profiling. J Immunol. 2007;179(6):3662-3671.

15. Laurent E, Doulatov $S$, Zandi S, et al. The transcriptional architecture of early human hematopoiesis identifies multilevel control of lymphoid commitment. Nat Immunol. 2013;14(7):756-763. Nature. 2015;517(7536):583-588.

haematological 2018; 103:e526 